\title{
Retinal macular dystrophy type 2
}

INSERM

\section{Source}

INSERM. (1999). Orphanet: an online rare disease and orphan drug data base. Retinal macular dystrophy type 2. ORPHA:319640

Retinal macular dystrophy type 2 is a rare, genetic macular dystrophy disorder characterized by slowly progressive "bull's eye" maculopathy associated, in most cases, with mild decrease in visual acuity and central scotomata. Usually, only the central retina is involved, however some cases of more widespread rod and cone anomalies have been reported. Rare additional features include empty sella turcica, impaired olfaction, renal infections, hematuria and recurrent miscarriages. 